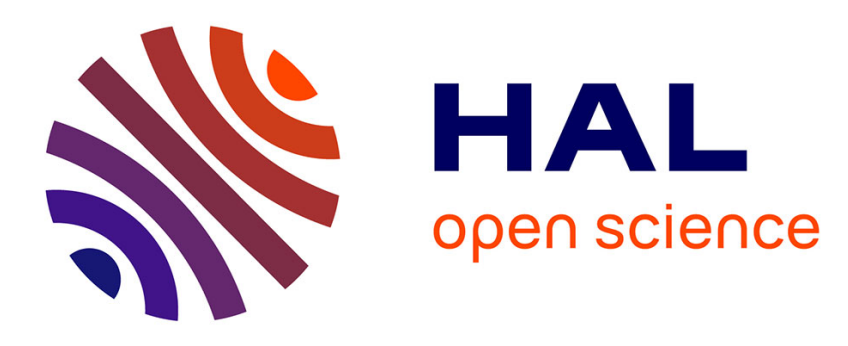

\title{
Finite Dimension Wyner-Ziv Lattice Coding for Two-Way Relay Channel
}

Sinda Smirani, Mohamed Kamoun, M. Sarkis, Abdellatif Zaidi, Pierre Duhamel

\section{- To cite this version:}

Sinda Smirani, Mohamed Kamoun, M. Sarkis, Abdellatif Zaidi, Pierre Duhamel. Finite Dimension Wyner-Ziv Lattice Coding for Two-Way Relay Channel. IEEE 14th Workshop on Signal Processing Advances in Wireless Communications (SPAWC 2013), Jun 2013, Darmstadt, Germany. pp.1-5, 10.1109/spawc.2013.6612114 . hal-00816670

\section{HAL Id: hal-00816670 \\ https://hal-centralesupelec.archives-ouvertes.fr/hal-00816670}

Submitted on 5 Oct 2018

HAL is a multi-disciplinary open access archive for the deposit and dissemination of scientific research documents, whether they are published or not. The documents may come from teaching and research institutions in France or abroad, or from public or private research centers.
L'archive ouverte pluridisciplinaire HAL, est destinée au dépôt et à la diffusion de documents scientifiques de niveau recherche, publiés ou non, émanant des établissements d'enseignement et de recherche français ou étrangers, des laboratoires publics ou privés. 


\section{Finite Dimension Wyner-Ziv Lattice Coding for Two-Way Relay Channel}

\author{
Sinda Smirani, Mohammed Kamoun, Mireille Sarkiss \\ CEA, LIST, Communicating Systems Laboratory \\ BC 94, Gif Sur Yvette, F91191 - France \\ \{sinda.smirani, mohamed.kamoun, mireille.sarkiss\}@cea.fr
}

\author{
Abdellatif Zaidi \\ Université Paris-Est Marne La Vallée \\ Champs-sur-Marne, F77454 - France \\ abdellatif.zaidi@univ-mlv.fr
}

\author{
Pierre Duhamel \\ CNRS/LSS, Supelec \\ Gif Sur Yvette, F91192 - France \\ pierre.duhamel@1ss.supelec.fr
}

\begin{abstract}
Two-way relay channel (TWRC) models a cooperative communication situation performing duplex transmission via a relay station. For this channel, we have shown previously that a lattice-based physical layer network coding strategy achieves, at the limit of arbitrarily large dimension, the same rate as that offered by the random coding-based regular compressand-forward. In this paper, we investigate a practical coding scheme using finite dimension lattices and offering a reasonable performance-complexity trade-off. The algorithm relies on lattice based quantization for Wyner-Ziv coding. We characterize the rate region allowed by our coding scheme, discuss the design criteria, and illustrate our results with some numerical examples.
\end{abstract}

Keywords: TWRC, compress and forward, Wyner-Ziv, lattice codes, finite dimension

\section{INTRODUCTION}

The two way relaying problem where two communicating nodes want to exchange information via a relay is encountered in various wireless communication scenarios: ad-hoc networks, range extension for cellular and local networks ... While network level routing is the standard option to this problem, it has been shown that network coding (NC) strategies provide better performance by leveraging the side information that is available in each node. In fact, NC allows to improve the rates by combining raw bits or packets at the network layer. The capacity of the system can be further improved when NC is applied to the physical layer. It takes advantage of the linear superposition properties of the wireless channel in order to turn interference nuisance into useful signal [1]. In this context, we consider a physical network coding (PNC) strategy where the overall communication takes two phases, namely a Multiple Access (MAC) phase and a Broadcast (BC) phase.

Various strategies have been proposed for TWRC. Amplify and Forward [2], Decode and Forward (DF) [3], and Compress and Forward. The latter has attracted particular attention since it offers a good compromise between processing complexity at the relay and noise amplification. CF for TWRC [4] follows the same strategy of CF schemes for the relay channel [5]. Performance bounds of this scheme have been investigated in [6], [7], [8]. It has been shown that for specific channel conditions, specially symmetric channels, CF outperforms the other relaying schemes for high SNR regimes. In the aforementioned references, the derivation of the achievable rate regions has employed high dimension assumptions and random coding approach which is impractical for real systems. Interestingly, structured codes have been found to be more advantageous in practical settings thanks to their reduced complexity in encoding and decoding [9]. It has been shown in [10] that for an Additive White Gaussian Noise (AWGN) channel, lattice codes can achieve the Shannon capacity for Gaussian pointto-point communication. Based on this result, lattice codes have been extended to TWRC scenario in [11] and [12] as follows : the transmitters employ nested lattices as codebooks, and the relay decodes a modulo-lattice sum of the transmitted codewords from the received signal in order to emulate a XOR operation at the packet level. All nodes (transmitters and relay) are constrained to transmit with the same power and consequently they use the same lattice codebook. In both schemes, the relay follows a DF strategy since it decodes a function of the transmitted lattice codewords. In this paper, we design a new relaying scheme for TWRC based on CF strategy where the relay only compresses the received signal from both nodes based on a knowledge of their transmit power and the channel gain modules. Unlike [11] and [12], our scheme employs lattice encoding only by the relay and is compatible with arbitrary transmit powers and channel gains.

In the MAC phase of our scheme, the communicating nodes send simultaneously their messages and the relay receives a mixture of the transmitted signals. The relay considers this mixture as a source which will be compressed and sent during the $\mathrm{BC}$ phase. Taking into account that each terminal has a partial knowledge of this source (side information), the BC phase is equivalent to a Wyner-Ziv (WZ) compression setting with two decoders having a piece of side information each. A lattice based lossy compression is employed to help each user generate a local distorted version of the source. The proposed scheme is based on lattice quantization introduced in [13] and which we extend to the TWRC case. In [14], we have showed that with infinite dimension lattices, this scheme achieves the same rates as the random coding compress and forward strategy. With finite dimension lattices, the decoding error probability cannot be arbitrarily small. Based on this observation, we derive, in this paper, achievable rate regions by considering non vanishing yet constrained decoding error probabilities. The rest of the paper is organized as follows. In 
section II, we introduce our system model. In section III and IV, we propose a new lattice-based Wyner-Ziv Coding scheme and we derive its achievable rate region for finite dimensions. In section $\mathrm{V}$, we present a numerical implementation of the achievable rates with practical finite dimension lattices. Finally, section VI concludes the paper.

Notations Random variables (r.v.) are indicated by capital letters where the realizations are written in small letters. Vector of r.v. or a sequence of realizations are indicated by bold fonts.

\section{SySTEM MODEL}
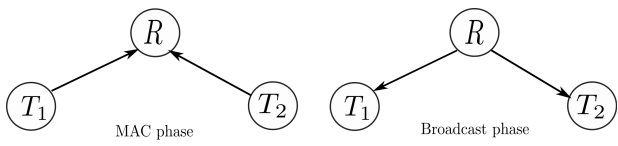

Fig. 1. The two-phase transmission of TWRC

We consider a Gaussian TWRC in which two nodes $T_{1}$ and $T_{2}$ exchange two individual messages $m_{1}$ and $m_{2}$, with the help of a relay $R$ as shown in Fig.1. The relay operates in halfduplex mode. The communication takes $n$ channel uses that are split among MAC phase and BC phase with lengths $n_{1}=$ $\alpha n$ and $n_{2}=(1-\alpha) n, \alpha \in[0,1]$ respectively. During the MAC phase, node $\mathrm{T}_{i}, i=1,2$ draws uniformly a message $m_{i}$ from the set $\mathcal{M}_{i}=\left\{1,2, \cdots, 2^{n R_{i \bar{i}}}\right\}$ and sends it to the other terminal. Let $\mathbf{x}_{i}\left(m_{i}\right)$ denotes the codeword of length $n_{1}$ sent by node $\mathrm{T}_{i}, i=1,2$. The messages are transmitted through a memoryless Gaussian channel and the relay $\mathrm{R}$ receives a signal $\mathbf{Y}_{\mathbf{r}}$.

During the BC phase, the relay generates a codeword $\mathbf{x}_{r}\left(m_{r}\right)$ of dimension $n_{2}$ from the received sequence $\mathbf{y}_{r}$. The signal $X_{r}$ is transmitted through a broadcast memoryless channel and the received signal at node $\mathrm{T}_{i}$ is $\mathbf{Y}_{\mathbf{i}}, i=1,2$.

All input distributions are real valued: $X_{k} \sim \mathcal{N}\left(0, P_{k}\right), k=$ $\{1,2, r\}$, where $\mathcal{N}\left(0, P_{k}\right)$ denotes a zero mean real Gaussian variable with power $P_{k}$. The received signals can be modeled as follows:

$$
\begin{aligned}
& Y_{r}=h_{1} X_{1}+h_{2} X_{2}+Z_{r} \\
& Y_{i}=h_{i} X_{r}+Z_{i},
\end{aligned}
$$

where $h_{i}$ denotes the channel coefficient between $\mathrm{T}_{i}$ and $\mathrm{R}$, $i=1,2$. Without loss of generality, channel reciprocity is assumed, i.e. $h_{i \rightarrow r}=h_{r \rightarrow i}=h_{i} . Z_{r} \sim \mathcal{N}\left(0, \sigma_{r}^{2}\right)$ is the additive white Gaussian noise at the relay and $Z_{i} \sim \mathcal{N}\left(0, \sigma_{i}^{2}\right)$ is the AWGN at node $\mathrm{T}_{i}, i=1,2$. We assume perfect CSI for all nodes and the noise components are independent of each other and from the channel inputs. In the sequel, we investigate the achievable rates and the design of our scheme.

\section{Achievable Rate Region FOR TWRC}

Theorem 3.1: Let $\left(\Lambda_{1}, \Lambda_{2}\right)$, a pair of two nested lattices of dimension $n_{1}$, with $\Lambda_{2} \subset \Lambda_{1}$. For Gaussian TWRC, the convex hull of the following end-to-end rates $\left(R_{12}, R_{21}\right)$ is achievable:

$$
\begin{aligned}
& R_{12} \leq \frac{\alpha}{2} \log _{2}\left(1+\frac{\left|h_{1}\right|^{2} P_{1}\left(\left|h_{1}\right|^{2} P_{1}+\sigma_{r}^{2}-D_{2}\right)}{\left|h_{1}\right|^{2} P_{1}\left(\sigma_{r}^{2}+D_{2}\right)+\sigma_{r}^{4}}\right) \\
& R_{21} \leq \frac{\alpha}{2} \log _{2}\left(1+\frac{\left|h_{2}\right|^{2} P_{2}\left(\left|h_{1}\right|^{2} P_{1}+\sigma_{r}^{2}-D_{2}\right)}{\left|h_{1}\right|^{2} P_{1}\left(\sigma_{r}^{2}+D_{2}\right)+\sigma_{r}^{4}}\right)
\end{aligned}
$$

where $D_{2}$ satisfies:

$$
\begin{array}{r}
\alpha\left(\log _{2}\left(\frac{\sigma_{U_{2}}^{2}}{D_{2}}\right)+\log _{2}\left(G\left(\Lambda_{1}\right) \mu\left(\Lambda_{2}\right)\right)\right) \leq \\
(1-\alpha) \min \left\{\log _{2}\left(1+\frac{\left|h_{2}\right|^{2} P_{r}}{\sigma_{2}^{2}}\right), \log _{2}\left(1+\frac{\left|h_{1}\right|^{2} P_{r}}{\sigma_{1}^{2}}\right)\right\}
\end{array}
$$

with $G\left(\Lambda_{1}\right)$ being the normalized second moment of $\Lambda_{1}$ and $\mu\left(\Lambda_{2}\right)$ being the volume to noise ratio of $\Lambda_{2}$ [15], and $\alpha \in$ $[0,1]$.

Remark 1: Letting $n_{1} \rightarrow \infty$, the left-hand side expression in (5) reduces to its first term since the second term corresponds to the penalty of using finite dimension, that vanishes asymptotically. We have shown in [14] that the achievable rate region coincides with the random coding compress and forward achievable rate region presented in [8].

\section{Proof of Theorem 3.1}

In this section, we present a detailed proof of theorem 3.1. The main idea of the proposed scheme is the following: during the $\mathrm{BC}$ phase, the relay station sends a compressed version of the signal received during the MAC phase. The relay employs a lossy compression Wyner-Ziv scheme using nested lattices that is tuned to the side information of the user with the weakest side information. The proof is divided into three paragraphs: in section IV-A, we present the WZ strategy based on the weakest side information at the receivers. In section IV-B, the lattice coding scheme for the WZ model is introduced and finally the achievable rates of the proposed scheme are derived in IV-C.

\section{A. Wyner-Ziv using the weakest side information}

Let $\mathbf{S}_{i}=h_{i} \mathbf{X}_{i}$ be the side information available at terminal $\mathrm{T}_{i}, i=1,2$. Without loss of generality, we assume that $\left|h_{2}\right|^{2} P_{2} \leq\left|h_{1}\right|^{2} P_{1}$. With this setting, $\mathrm{T}_{2}$ is the terminal who experiences the weakest side information. The quantization performed by the relay is tuned so that $\mathrm{T}_{2}$ reconstructs a local version $\hat{\mathbf{Y}}_{r, i}$ of $\mathbf{Y}_{r}$ with a distortion $D_{2}: \frac{1}{n_{1}} \mathbb{E}\left\|\mathbf{Y}_{r}-\hat{\mathbf{Y}}_{r, 2}\right\|^{2} \leq$ $D_{2}$. The terminal $\mathrm{T}_{1}$ will undergo this choice on its decoded signal at the end of transmission.

The source $\mathbf{Y}_{r}$ can be written as the sum of two independent Gaussian r.v.: the side information $\mathbf{S}_{2}$ and the unknown part $\mathbf{U}_{2}=\mathbf{Y}_{r} \mid \mathbf{S}_{2}=h_{1} \mathbf{X}_{1}+\mathbf{Z}_{r}$ that will be decoded at the end. The variance per dimension of $\mathbf{U}_{2}$ is $\sigma_{U_{2}}^{2}=\operatorname{VAR}\left(Y_{r} \mid S_{2}\right)=$ $\left|h_{1}\right|^{2} P_{1}+\sigma_{r}^{2}$.

\section{B. Lattice based source coding}

We use a pair of $n_{1}$-dimensional nested lattices $\left(\Lambda_{1}, \Lambda_{2}\right)$ chosen as in [13]: the fine lattice $\Lambda_{1}$ is good for quantization with basic Voronoi region $\mathcal{V}_{1}$ of volume $V_{1}$ and second 
moment per dimension $\sigma^{2}\left(\Lambda_{1}\right)=D_{2}$ and the coarse lattice $\Lambda_{2}$ is good for channel coding with basic Voronoi region $\mathcal{V}_{2}$ of volume $V_{2}$ and second moment $\sigma^{2}\left(\Lambda_{1}\right)=\sigma_{U_{2}}^{2}$.

The encoding operation is performed with four successive operations: first, the input signal $\mathbf{Y}_{r}$ is scaled with a factor $\beta$. Then, a random dither which is uniformly distributed over $\mathcal{V}_{1}$ is added. This dither is known by all nodes. The dithered scaled version of $\mathbf{Y}_{r}, \beta \mathbf{y}_{r}+\mathbf{t}$ is quantized to the nearest point in $\Lambda_{1}$. The outcome of this operation is processed with a modulo-lattice operation in order to generate a vector $\mathbf{v}_{r}$ of size $n_{1}$ as shown in Fig. 2 .

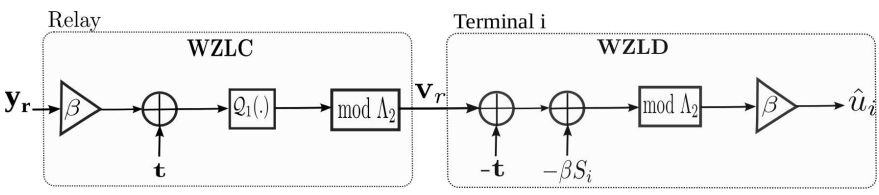

Fig. 2. WZ Lattice Coding and Decoding at $\mathrm{T}_{i}, i=1,2$

$$
\mathbf{v}_{r}=Q_{1}\left(\beta \mathbf{y}_{r}+\mathbf{t}\right) \bmod \Lambda_{2}
$$

The relay sends the index of $\mathbf{v}_{r}$ that identifies the coset of $\Lambda_{2}$ relative to $\Lambda_{1}$ that contains $Q_{1}\left(\beta \mathbf{y}_{r}+\mathbf{t}\right)$. The coset leader $\mathbf{v}_{r}$ is represented with $\frac{V_{2}}{V_{1}}$. bits. Thus, the source coding rate of the scheme is

$R\left(D_{2}\right)=\frac{1}{n_{1}} \log _{2}\left|\Lambda_{1} \cap \mathcal{V}_{2}\right|=\frac{1}{n_{1}} \log _{2} \frac{V_{2}}{V_{1}}$ (bits per dimension)

At both users, $\mathbf{v}_{r}$ is decoded first. Then $\hat{\mathbf{Y}}_{r, i} \mid \hat{\mathbf{S}}_{i}=\hat{\mathbf{U}}_{i}$ is reconstructed with a WZ lattice decoder (WZLD) using the side information $\mathbf{S}_{i}$ as

$$
\hat{\mathbf{u}}_{i}=\beta\left(\left(\mathbf{v}_{r}-\mathbf{t}-\beta \mathbf{s}_{i}\right) \quad \bmod \Lambda_{2}\right), i=1,2
$$

\section{Rate analysis}

At the relay, the message $m_{r}$ corresponding to the index of $\mathbf{v}_{r}$ is mapped to a codeword $\mathbf{x}_{r}$ of size $n_{2}$. Let $R_{r}$ be the common broadcast rate. This rate is bounded by

$$
n_{1} R\left(D_{2}\right) \leq n_{2} R_{r}
$$

On the other hand,

$$
R_{r} \leq \min \left(I\left(X_{r} ; Y_{1}\right), I\left(X_{r} ; Y_{2}\right)\right)
$$

Since real Gaussian codebooks are used for all transmissions, we have: $I\left(X_{r} ; Y_{i}\right)=\frac{1}{2} \log _{2}\left(1+\frac{\left|h_{i}\right|^{2} P_{r}}{\sigma_{i}^{2}}\right), i=1,2$. This constraint ensures that the index $m_{r}$ is transmitted reliably to both terminals and $\mathbf{v}_{r}$ is available at the input of WZLD at both receivers. At terminal $\mathrm{T}_{2}, \hat{\mathbf{u}}_{2}$ in (8) can be written as:

$$
\begin{aligned}
\hat{\mathbf{u}}_{2} & =\beta\left(\left(\beta \mathbf{u}_{2}+\mathbf{e}_{q}\right) \bmod \Lambda_{2}\right) \\
& \equiv \beta\left(\beta \mathbf{u}_{2}+\mathbf{e}_{q}\right)
\end{aligned}
$$

where $\mathbf{e}_{q}=Q_{1}\left(\beta \mathbf{y}_{r}+\mathbf{t}\right)-\left(\beta \mathbf{y}_{r}+\mathbf{t}\right)=-\left(\beta \mathbf{y}_{r}+\mathbf{t}\right) \bmod \Lambda_{1}$, is the quantization error. By the Crypto Lemma [10], $\mathbf{E}_{q}$ is independent from $\mathbf{Y}_{r}$, thus $\mathbf{U}_{2}$, and it is uniformly distributed over $\mathcal{V}_{1}$ i.e $\operatorname{VAR}\left(E_{q}\right)=\sigma^{2}\left(\Lambda_{1}\right)=D_{2}$. The equivalence between (11) and (12) is valid only if $\beta \mathbf{u}_{2}+\mathbf{e}_{q} \in \mathcal{V}_{2}$. With finite dimension lattices, the volume of $\mathcal{V}_{2}$ should be large enough to enclose this signal. In this case, provided that

$$
\frac{1}{n_{1}} \mathbb{E}\left\|\mathbf{E}_{q}+\beta \mathbf{U}_{2}\right\|^{2}=D_{2}+\beta^{2} \sigma_{U_{2}}^{2} \leq \sigma^{2}\left(\Lambda_{2}\right)
$$

The rates are calculated by ensuring that the probability $\operatorname{Pr}\left(\beta \mathbf{U}_{2}+\mathbf{E}_{q} \notin \mathcal{V}_{2}\right)$ does not exceed a fixed threshold.

$$
\operatorname{Pr}\left(\beta \mathbf{U}_{2}+\mathbf{E}_{q} \notin \mathcal{V}_{2}\right) \leq P_{e}
$$

Given that $V_{1}=\left(\frac{\sigma^{2}\left(\Lambda_{1}\right)}{G\left(\Lambda_{1}\right)}\right)^{n_{1} / 2}$ where $G\left(\Lambda_{1}\right)$ is the normalized second moment (NSM) of $\Lambda_{1}$ and $\sigma^{2}\left(\Lambda_{1}\right)=D_{2}$, the coding rate in (7) reads:

$$
R\left(D_{2}\right)=\frac{1}{2} \log _{2}\left(\frac{\sigma_{U_{2}}^{2}}{D_{2}}\right)+\frac{1}{2} \log _{2}\left(G\left(\Lambda_{1}\right) \mu\left(\Lambda_{2}\right)\right)
$$

The WZ rate distorsion function is achieved with a redundancy term $L=\frac{1}{2} \log _{2}\left(G\left(\Lambda_{1}\right) \mu\left(\Lambda_{2}\right)\right)$, where

$$
\mu\left(\Lambda_{2}\right)=\frac{V_{2}^{\frac{2}{n_{1}}}}{\sigma_{U_{2}}^{2}}
$$

is the $\Lambda_{2}$ volume to noise ratio (VNR) associated with probability of error $P_{e}$. This term has been introduced by Poltyrev in [16] for lattice codes in AWGN setting. For a probability $P_{e}$ and a lattice $\Lambda$ with volume $V, \mu(\Lambda)=V^{\frac{2}{n_{1}}} / \sigma^{2}, \sigma^{2}$ is the variance of a Gaussian noise $\mathbf{Z}$ which verifies $\operatorname{Pr}(\mathbf{Z} \notin$ $\mathcal{V}) \leq P_{e}$. By analogy to our problem, taking into account the constraints expressed in (13) and (14), the VNR is given by (16). Finally, (5) is obtained by combining equations (9), (10) and (15).

The parameter $\beta$ has to be chosen so that to verify (13) and (17).

$$
\frac{1}{n_{1}} \mathbb{E}\left\|\mathbf{Y}_{r}-\hat{\mathbf{Y}}_{r, 2}\right\|^{2}=\left(1-\beta^{2}\right)^{2} \sigma_{U_{2}}^{2}+\beta^{2} D_{2} \leq D_{2}
$$

Thus, the optimal scaling factor $\beta$ is $\beta=\sqrt{1-\frac{D_{2}}{\sigma_{U_{2}}^{2}}}$ (see [13]). By replacing $\mathbf{U}_{2}$ by its value we conclude that:

$$
\hat{\mathbf{U}}_{2}=\beta^{2} h_{1} \mathbf{X}_{1}+\beta^{2} \mathbf{Z}_{r}+\beta \mathbf{E}_{q}
$$

Let $\mathbf{Z}_{e q}=\beta^{2} \mathbf{Z}_{r}+\beta \mathbf{E}_{q}$ be the effective additive noise. The communication between $\mathrm{T}_{1}$ and $\mathrm{T}_{2}$ is equivalent to a virtual additive Gaussian channel where the noise is given by $\mathbf{Z}_{e q}$. Let $\mathbf{Z}_{q}$ a Gaussian variable with same variance as $\mathbf{E}_{q}$. Based on the results in [17], we have

$\mathcal{D}\left(\beta^{2} U_{2}+\beta E_{q}, \beta^{2} U_{2}+\beta Z_{q}\right)=h\left(\beta^{2} U_{2}+\beta Z_{q}\right)-h\left(\beta^{2} U_{2}+\beta E_{q}\right)$

where $\mathcal{D}(.,$.$) is the relative entropy. Let \lambda=\frac{D_{2}}{\sigma_{U_{2}}^{2}}, M=\frac{E_{q}}{\sqrt{\lambda}}$ and $M^{*}=\frac{Z_{q}}{\sqrt{\lambda}}$, we can verify that $h\left(\beta^{2} U_{2}+\beta E_{q}\right)=h\left(\sqrt{\lambda} M+\sqrt{1-\lambda} M^{*}\right)$ 
Since $U_{2}$ is Gaussian, this entropy increases to zero monotonically as $D_{2}$ goes from zero to $\sigma_{U_{2}}^{2}$ as shown in [17]. Equivalently for $D_{2} \rightarrow 0$, the entropy increases to $h\left(M^{*}\right)$ :

$$
\begin{aligned}
h\left(\beta^{2} U_{2}+\beta E_{q}\right) & \rightarrow h\left(M^{*}\right)=h\left(Z_{q}\right)-\log _{2}(\sqrt{\lambda}) \\
& =\frac{1}{2} \log _{2}\left(2 \pi e D_{2}\right)-\frac{1}{2} \log _{2}\left(\frac{D_{2}}{\sigma_{U_{2}}^{2}}\right)
\end{aligned}
$$

We have $h\left(\beta^{2} U_{2}+\beta Z_{q}\right)=\frac{1}{2} \log _{2}\left(2 \pi e \sigma_{U_{2}}^{2}\right)$. Thus from (19),

$$
\begin{aligned}
D\left(\hat{U}_{2}, \beta^{2} U_{2}+\beta Z_{q}\right) & \rightarrow-\frac{1}{2} \log _{2}\left(\frac{D_{2}}{\sigma_{U_{2}}^{2}}\right)+\frac{1}{2} \log _{2}\left(\frac{D_{2}}{\sigma_{U_{2}}^{2}}\right) \\
& =0
\end{aligned}
$$

We conclude that in the divergence sense, for high resolution assumption as $D_{2} \rightarrow 0$, we can approximate the decoded signal with a Gaussian one. Thus the achievable rate of this link satisfies:

$$
n R_{12} \leq \frac{n_{1}}{2} \log _{2}\left(1+\frac{\beta^{2}\left|h_{1}\right|^{2} P_{1}}{\beta^{2} \sigma_{r}^{2}+D_{2}}\right)
$$

by replacing $\frac{n_{1}}{n}=\alpha$ and $\beta$ by its value, (3) is verified.

At terminal $\mathrm{T}_{1}^{n}$, the decoder is tailored to the side information $\mathbf{S}_{1}$. Thus, at the decoder we subtract $\beta \mathbf{s}_{1}$ and $\hat{\mathbf{u}}_{1}$ is reconstructed similarly to $\hat{\mathbf{u}}_{2}$ in (11). Since $\sigma_{S_{1}}^{2} \geq \sigma_{S_{2}}^{2}$, we have

$$
\begin{aligned}
\sigma_{U_{1}}^{2} & \leq \sigma_{U_{2}}^{2}, \\
\operatorname{Pr}\left(\beta \mathbf{U}_{1}+\mathbf{E}_{q} \notin \mathcal{V}_{2}\right) & \leq \operatorname{Pr}\left(\beta \mathbf{U}_{2}+\mathbf{E}_{q} \notin \mathcal{V}_{2}\right), \\
D_{1}=\frac{1}{n_{1}} \mathbb{E}\left\|\mathbf{Y}_{r}-\hat{\mathbf{Y}}_{r, 1}\right\|^{2} & \leq D_{2}
\end{aligned}
$$

The communication between $T_{2}$ and $T_{1}$ is equivalent to a virtual Gaussian channel with an additive noise $\mathbf{Z}_{e q}$ and a rate:

$$
n R_{21} \leq \frac{n_{1}}{2} \log _{2}\left(1+\frac{\beta^{2}\left|h_{2}\right|^{2} P_{2}}{\beta^{2} \sigma_{r}^{2}+D_{2}}\right)
$$

which verifies (4) and concludes the proof. The whole coding scheme is summarized in Fig.3.

Remark 2: It is possible to use $\mathbf{S}_{1}$ as the side information for the WZ lattice coding scheme to achieve a controlled distortion $D_{1}$ at terminal $\mathrm{T}_{1}$. For this purpose, we need two coding layers: a common layer to be sent to both nodes and a refinement layer to be decoded only by the best node $\mathrm{T}_{1}$. In this case, the achievable rates can be ameliorated. This study is under investigation.

\section{NUMERICAL IMPLEMENTATION}

In this section, we present the achievable rates for practical finite dimensional lattices. In this case, a rate loss is incurred in the coding rate comparing to the $\mathrm{WZ}$ rate distortion function as described in previous sections (III and IV). The achievable rate region is calculated by ensuring that the error probability $\operatorname{Pr}\left(\beta \mathbf{U}_{2}+\mathbf{E}_{q} \notin \mathcal{V}_{2}\right) \leq P_{e}$. The analytical derivation of the error probability for practical lattice pairs is difficult in general since it requires the integration over the Voronoi region of the coarse lattice. Though it can be computed numerically using Monte Carlo integration or approximated by an upper bound.
TABLE I

SOME IMPORTANT BINARY LATTICES AND THEIR USEFUL PROPERTIES

\begin{tabular}{|c|l|l|l|l|}
\hline Lattice $\Lambda$ & dimension $n_{1}$ & $G(\Lambda)$ & $\gamma_{c}(\Lambda)$ & $K(\Lambda)$ \\
\hline \hline $\mathbb{Z}^{k}$ & $k$ & 0.0833 & 1 & $2 k$ \\
\hline$D_{4}$ & 4 & 0.07660 & $\sqrt{2}$ & 24 \\
\hline$E_{8}$ & 8 & 0.071682 & 2 & 240 \\
\hline$\Lambda_{16}$ & 16 & 0.06829 & $2^{3 / 2}$ & 4320 \\
\hline$\Lambda_{24}$ & 24 & 0.00657 & 4 & 196560 \\
\hline
\end{tabular}

An approximation of the error probability can be obtained using union bound and Chernoff bound:

$$
\operatorname{Pr}\left(\beta \mathbf{U}_{2}+\mathbf{E}_{q} \notin \mathcal{V}_{2}\right) \approx K\left(\Lambda_{2}\right) \exp \left(-\frac{1}{8} \gamma_{c}\left(\Lambda_{2}\right) \mu\left(\Lambda_{2}, P_{e}\right)\right)
$$

for sufficiently large $\mu\left(\Lambda_{2}, P_{e}\right) \cdot \gamma_{c}\left(\Lambda_{2}\right)=\frac{d_{\min }^{2}\left(\Lambda_{2}\right)}{V\left(\Lambda_{2}\right)^{2 / n_{1}}}$ is the coding gain of $\Lambda_{2}$ with $d_{\min }\left(\Lambda_{2}\right)$ is the minimum distance between two points in $\Lambda_{2}$. We choose the VNR in (15) as follows

$$
\mu\left(\Lambda_{2}, P_{e}\right) \approx \frac{8}{\gamma_{c}\left(\Lambda_{2}\right)} \log _{2}\left(\frac{K\left(\Lambda_{2}\right)}{P_{e}}\right)
$$

Note that $\mu\left(\Lambda_{2}, P_{e}\right) \gg \frac{1}{\gamma_{c}\left(\Lambda_{2}\right)}$ for small error probability. This guarantees that the union bound approximation is valid and the error probability is upper bounded by $P_{e}$.

Furthermore, given that the error probability of the scheme is defined by the goodness of the coarse lattice, the performance of the end to end scheme depends more on this lattice rather than the choice of the fine lattice. Therefore, the simple cubic lattice $\mathbb{Z}^{n_{1}}$ with NSM $G\left(\Lambda_{1}\right)=\frac{1}{12}$ will be the preferred choice for the fine lattice. In this case, for a good coarse lattice with NSM $G\left(\Lambda_{2}\right)=\frac{1}{2 \pi e}$, the rate loss with respect to the ideal WZ scheme is only $\frac{1}{2} \log _{2}(2 \pi e / 12)=0.2546$ bit per sample. Moreover, in the quantization problem, the choice of the fine (resp. coarse) lattice is equivalent to the choice of the coarse (resp. fine) lattice for the dual channel coding problem. It has been shown in [18] that practically $\mathbb{Z}^{n_{1}}$ suffices as a shaping lattice that verifies arbitrary small error probability. Thus, a sublattice of $\mathbb{Z}^{n_{1}}$ can be a simple engineering choice for the fine lattice. In the sequel, $\Lambda_{1}$ is a scaled version of $\mathbb{Z}_{1}^{n}$ i.e. $\Lambda_{1}=\eta \mathbb{Z}^{n_{1}}$.

We characterize the whole achievable rate region of the proposed scheme by optimizing the time division $\alpha \in[0,1]$ between MAC and $\mathrm{BC}$ phases and the distortion choice at the relay. The boundary bounds are determined by maximizing the weighted sum of both rates $R_{12}$ and $R_{21}$. We solve the following problem for $\theta \in[0,1]$

$$
\begin{array}{cc}
\max & \theta R_{12}+(1-\theta) R_{21} \\
\text { s.t. } & \left(R_{12}, R_{21}\right) \text { satisfy (3) and (4) } \\
& D_{2} \text { satisfies (5) for } \alpha \in[0,1]
\end{array}
$$

Table I gives the kissing number and the coding gain for a set of commonly used finite lattices, that can be used to calculate $\mu\left(\Lambda_{2}, P_{e}\right)$ for fixed $P_{e}$ using (21). Comparison between lattice pairs can be found in Fig. 4 for symmetric static channels, 


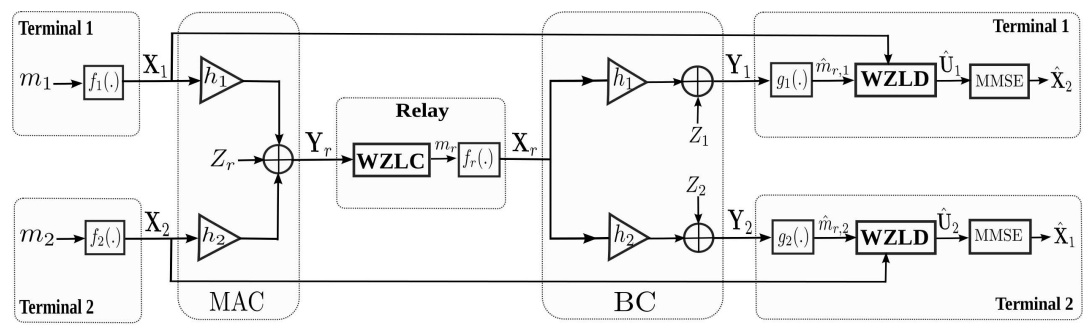

Fig. 3. Wyner-Ziv lattice-based coding scheme for TWRC

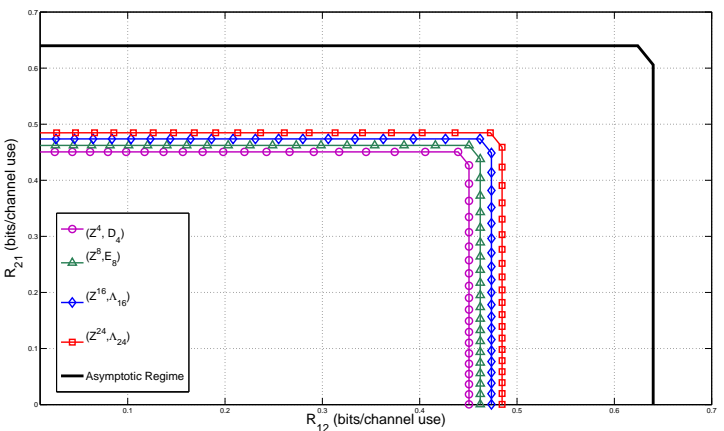

Fig. 4. Achievable rate region of different finite dimension lattice pairs compared to the high dimension achievable region for $\mathrm{SNR}_{1}=\mathrm{SNR}_{2}=$ $\mathrm{SNR}_{r}=10 \mathrm{~dB}$. A difference of $0.15 \mathrm{bit} /$ dimension between infinite and finite dimension lattices

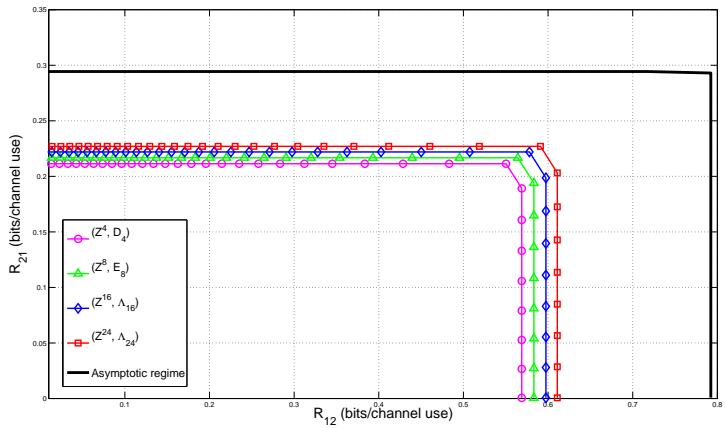

Fig. 5. Achievable rate region of different finite dimension lattice pairs compared to the high dimension achievable region for $\mathrm{P}_{1}=8 \mathrm{~dB}, \mathrm{P}_{2}=5 \mathrm{~dB}$, $\mathrm{P}_{r}=10 \mathrm{~dB},\left|h_{1}\right|=2$ and $\left|h_{2}\right|=1$

equal SNRs and $P_{e}=10^{-5}$. We notice that the difference between the infinite scheme and the pair $\left(\mathbb{Z}^{4}, \Lambda_{24}\right)$ is about $0.15 \mathrm{bit} / \mathrm{channel}$ use. In Fig. 5, we present the achievable rates for asymmetric channels and different power constraints. The gap between the infinite and finite dimensions is 0.2 bit/dimension for $R_{12}$ and $0.06 \mathrm{bit} /$ dimension for $R_{21}$. This indicates that by using practical lattices, the loss in rates according to the high dimensional regime $(n \rightarrow \infty)$ is significantly small.

\section{CONCLUSION}

In this paper, we derived a new achievable rate region for TWRC with finite dimension. We proposed for this purpose a new practical lattice-based physical layer network coding scheme. The scheme is based on Wyner-Ziv source coding strategy and nested lattice codes. We presented a numerical implementation of the achievable rates with practical finite dimension lattices.

\section{REFERENCES}

[1] S. Zhang, S. Liew, and P. Lam, "Physical layer network coding," in ACM MOBICOM, Los Angeles, USA, 2006.

[2] B. Rankov and A. Wittneben, "Spectral efficient signaling for halfduplex relay channels," in Asilomar Conference on Signals, Systems and Computers (ACSSC), Asilomar, CA, Nov. 2005.

[3] S. Smirani, M. Kamoun, and P. Duhamel, "Physical network coding for TWR channel: Capacity region and error exponents," in IFIP Wireless Days, Niagara Falls, Canada, Oct. 2011.

[4] B. Rankov and A. Wittneben, "Achievable rate regions for the twoway relay channel," in IEEE International Symposium on Information Theory, Seattle, Jul. 2006.

[5] T. M. Cover and A. E. Gamal, "Capacity theorems for the relay channel," IEEE Transactions on Information Theory, vol. 25, no. 5, pp. 572-584, Sep. 1979.

[6] C. Schnurr, T. J. Oechtering, and S. Stanczak, "Achievable rates for the restricted half-duplex two-way relay channel," in 41st Asilomar Conference on Signals, Systems and Computers (ACSSC), Asilomar, CA, Nov. 2007.

[7] S. J. Kim, N. Devroye, P. Mitran, and V. Tarokh, "Comparison of bidirectional relaying protocols," in IEEE Sarnoff Symposium, Princeton, NJ, Apr. 2008.

[8] D. Gunduz, E. Tuncel, and J. Nayak, "Rate regions for the separated two-way relay channel," in 46th Annual Allerton Conf. Comm. Control Computing, Illinois, Sep. 2008, p. 13331340.

[9] J. H. Conway and N. J. Sloane, Sphere packings, lattices and groups. New York, USA: Springe-Verlag, 3rd ed., 1998.

[10] U. Erez and R. Zamir, "Achieving $\frac{1}{2} \log (1+\mathrm{SNR})$ on the AWGN channel with lattice encoding and decoding," IEEE Transactions on Information Theory, vol. 50, no. 10, pp. 2293-2314, Oct. 2004.

[11] K. Narayanan, M. P. Wilson, and A. Sprintson, "Joint physical layer coding and network coding for bidirectional relaying," in 45th Annual Allerton Conf. Comm. Control Computing, Monticello, IL, Sep. 2007.

[12] W. Nam, S.-Y. Chung, and Y. H. Lee, "Capacity of the gaussian twoway relay channel to within $\frac{1}{2}$ bit," IEEE Transactions on Information Theory, vol. 56, pp. 5488-5494, Nov. 2010.

[13] R. Zamir, S. Shamai, and U. Erez, "Nested linear/lattice codes for structured multiterminal binning," IEEE Transactions on Information Theory, vol. 48, no. 6, pp. 1250 - 1276, Jun. 2002.

[14] S. Smirani, M. Kamoun, M. Sarkiss, A. Zaidi, and P. Duhamel, "Wynerziv lattice coding for two-way relay channel," in ATC 2012, Hanoi, Vietnam, Oct. 2012

[15] R. Zamir, "Lattices are everywhere," in 4th Annual Workshop on Information Theory and its Applications (ITA 2009), La Jolla, CA, Feb. 2009.

[16] G. Poltyrev, "On coding without restrictions for the awgn channel," IEEE Transactions on Information Theory, vol. 40, no. 52, pp. 409-417, Mar. 1994.

[17] R. Zamir and M. Feder, "Information rates of pre/post-filtered dithered quantizers," IEEE Transactions on Information Theory, vol. 42, no. 4, p. 13401353, Sep. 1996.

[18] G. D. F. Jr., M. D. Trott, and S. Y. Chung, "Sphere-bound-achieving coset codes and multilevel coset codes," IEEE Transactions on Information Theory, vol. 46, no. 3, p. 820850, May 2000. 